\title{
SEVERE INTESTINAL FAILURE IN A CHAGAS'S DISEASE PATIENT AFTER TOTAL COLECTOMY PLUS PARTIAL ILEECTOMY
} Thaís Castro Duarte', Fernanda Godoi Melo', José Rodrigues Santos-Júnior ${ }^{2}$, Marcel Pereira Moussa²,
Daurea Abadia De-Souza ${ }^{2}$, Multidisciplinary Residency Program in Clinical Nutrition

Corresponding author: Daurea Abadia De-Souza - daureas@ufu.br ${ }^{1} M S c$ in Health Sciences. Postgraduate Program in Health Sciences and Multidisciplinary Residency Program in Clinical Nutrition, Multidisciplinary Residency in Health. Faculty of Medicine at the Federal University of Uberlândia. Uberlândia, Minas Gerais, Brazil. ${ }^{2}$ MD. Medical Resident in the Internal Medicine Program, Faculty of Medicine, Federal University of Uberlândia. Uberlândia, Minas Gerais, Brasil. ${ }^{3}$ Full Professor, Internal Medicine Department. Postgraduate Program in Health Sciences. Coordinator of the Multidisciplinary Residency Program in Clinical Nutrition, Multidisciplinary Residency in Health. Faculty of Medicine at the Federal University of Uberlêndia. Uberlândia, Minas Gerais, Brazil. http://orcid.org/0000-0001-8314-5018

\begin{abstract}
Introduction: To report the clinical course of a Chagas's disease patient with severe intestinal failure after resection of the total colon and terminal ileum. Case Report: The patient underwent rectosigmoidectomy (of the sigmoid volvulus, in December 2009) and total colectomy plus partial ileectomy (May 2011). Patient underwent multiple hospitalizations caused by severe diarrhea (up to 23 stools/day), hydroelectrolytic disturbance and acute renal failure, severe proteinenergy malnutrition [loss of $34.9 \%$ of usual body weight (UBW)], and multiple episodes of sepsis. We prescribed parenteral nutrition solutions exclusively or concurrently with very small volumes (e.g., $40 \mathrm{ml} /$ day) of semi-elemental diet with low fat and high protein. After several weeks predetermined amounts of carbohydraterich foods (potatoes, rice, pasta, cassava), vegetables (chayote, carrots), low-fat meat, cream crackers, coconut water and fruit (watermelon, melon, banana) were progressively introduced. After 36 months, the patient was metabolically stable ( $B W=67.2 \mathrm{~kg}$, weight recovery of $23.6 \mathrm{~kg}$ ), with hydroelectrolytic balance. Due to the recovery of the functional capacity, the patient was advised to return to his work activities. Conclusions: Chagas's disease patient submitted to intestinal resection may have a severe intestinal failure and protein-energy malnutrition. Specialized nutritional therapy and clinical and laboratory monitoring by a multidisciplinary team, may contribute to better prognostic.
\end{abstract}

Keywords: Intestinal resection; protein-energy malnutrition; diarrhea; fluid and electrolyte imbalance; systemic infection. 


\section{INTRODUCTION}

Intestinal failure is defined as the reduction of gut function below the minimum necessary for the absorption of macronutrients and/or water and electrolytes, such that intravenous supplementation is required to maintain health and/or growth'.

According to European Society for Clinical Nutrition and Metabolism (ESPEN), patients with intestinal failure can be functionally classified on the basis of onset, metabolic and expected outcome criteria'.

The most common cause of intestinal failure is short bowel syndrome (SBS). The definition of SBS is controversial. Some investigators describe SBS in anatomical terms $(<200 \mathrm{~cm}$ of functional small intestine $)^{2}$, while others have suggested that SBS should be defined according to the functional capacity of the bowel ${ }^{3}$. A functional SBS may develop in patients with several clinical conditions characterized by impairment in intestinal absorption, such as smaller resection of the small intestine with or without simultaneous resection of the large bowel, chronic intestinal obstruction or pseudoobstruction (e.g., Chagas' disease megacolon), Crohn's disease, radiation enteritis, or refractory sprue ${ }^{2}$.

From a functional standpoint, the human gastrointestinal tract is not uniform. The intact gastrointestinal tract exhibits a proximal-to-distal anatomic absorptive gradient, with anatomical specificities of the intestinal segments concerning the absorption of specific nutrients. This anatomic gradient is directly related to the functional capacity of the gastrointestinal tract ${ }^{4,5}$. When the gastrointestinal tract is intact, the colon plays a prominent role in maintaining fluid and electrolyte balance and healthy nutritional status ${ }^{6}$.

The patient's risk of losing nutritional autonomy (i.e., independence from intravenous nutrition) is directly related to the length of bowel remaining after bowel resection, as well as the primary intestinal disease ${ }^{3}$. In addition, the configuration of the remaining bowel, namely whether there was any ileum or colon in continuity and the presence of the ileocecal valve, also affected the prognosis ${ }^{3}$. In patients with SBS, the colon becomes an important digestive organ responsible for the bacterial fermentation of carbohydrates and proteins that are not absorbed in the small intestine ${ }^{6}$. Indeed, patients undergoing small bowel resection plus total colectomy could present considerable increases in the loss of energy and nutrients through the feces ${ }^{7}$.

Although intestinal failure associated with functional SBS is not a direct determinant of mortality, the pathophysiological changes associated with this uncommon condition can lead to serious complications. In patients submitted to gastrointestinal surgery, longterm survival is directly affected by postoperative complications $^{8}$. Clinically, these patients could present septic, metabolic and complex nutritional complications. Fluid and electrolyte imbalance and renal impairment also may be present ${ }^{3,5}$.

Here, we report the clinical course of a patient with a functional short bowel syndrome characterized by severe intestinal failure, caused by total colectomy plus partial ileectomy, indicated for the surgical treatment of Chagas' disease intestinal complications. Severe protein-energy malnutrition, fluid, and electrolyte imbalance, and septic complications developed after surgical procedures, as well as the therapeutic procedures adopted were emphasized. The study was approved by the local Research Ethics Committee (CAAE 10260112.1.0000.5152) and the participant gave written informed consent.

\section{CASE REPORT}

A 57-year-old male Chagas' disease carrier with megacolon, presented sigmoid volvulus in December/2009, was admitted to the hospital and submitted to rectosigmoidectomy and colostomy. During hospitalization, the patient lost $9 \mathrm{~kg}$ body weight (BW) [usual $B W(u B W)=67 \mathrm{~kg}$ to $B W=58$ $\mathrm{kg}$, resulting in a reduction in body mass index ( $B M$; $B M I=22.4 \mathrm{~kg} / \mathrm{m} 2$ to $B M I=19.4 \mathrm{~kg} / \mathrm{m} 2$ ) and a weight loss percentage (WL\%) of $13.4 \%$ (Fig. 1). Due to the high output ostomy and diagnosis of proteinenergy malnutrition the patient received intravenous parenteral nutrition (PN) solutions for 16 days. 
With clinical improvement, PN was suspended and constipating, lactose and insoluble fiber-restricted oral diet was prescribed. After 25 days, the patient was discharged with instructions to follow-up in the outpatient clinic and for to use constipating oral diet in combination with partial volumes of polymeric enteral diet, high protein, oral route.

In May 2011, the patient was hospitalized for intestinal transit reconstruction. At that time, the patient presented preserved functional capacity and a relatively stable BW (a loss of $1.0 \mathrm{~kg}$ in 17 months). During the surgery, diffuse colonic dilatation was identified, and a total colectomy and partial ileectomy (removal of $60 \mathrm{~cm}$ of the terminal ileum and ileocecal valve) with ileorectal anastomosis were performed. The patient had an uneventful recovery and was discharged after six days of hospitalization.

Two days after discharge, the patient was readmitted with a diagnosis of fluid and electrolyte imbalance and acute renal failure caused by 23 episodes of large quantities of liquid stools/day. Upon discharge, the patient had fluid and electrolyte balance, normal renal function, partial improvement in the rate of evacuations, and $B W=53.2 \mathrm{~kg}$ (WL\% $=20.6$ compared with the UBW).

During multidisciplinary outpatient follow-up, the patient maintained a frequency of three to five liquid or pasty stools/day, with progressive loss of BW (47 kg, WL\%=29.9 compared to UBW). Within four months, the patient was readmitted for emergency treatment three times because of fluid and electrolyte imbalance and acute renal failure.

In September 2011 , the patient was again admitted with fluid and electrolyte imbalance $(\mathrm{Na}=124$ $\mathrm{mEq} / \mathrm{l}, \mathrm{K}=4.1 \mathrm{mEq} / \mathrm{l}, \mathrm{Mg}=0.7 \mathrm{mg} / \mathrm{dl}$ ), acute renal failure (urea $=108 \mathrm{mg} / \mathrm{dl}$, creatinine $=3.6 \mathrm{mg} / \mathrm{dl}$ ), and severe protein-energy malnutrition $(B W=44.7$ $\mathrm{kg} ; \mathrm{WL} \%=33.3 \%$ compared with the uBW). During hospitalization, the patient had several episodes of bacteremia andsepsis, withblood orcathetertipculture positive for Gram-positive cocci (Staphylococcus aureus) and Gram-negative (Pseudomonas aeruginosa and Klebsiella pneumoniae). The patient was treated with Metronidazole + Ciprofloxaxin (10 days), Teicoplanin (14 days), Imipenem (15 days), Trimethoprim + Sulphamethoxazole (14 days), Cefepime + Fluconazole, and Ceftriaxone (14 days). On this admission, the patient had the lowest $B W$ registered during his evolution ( $B W=43.6 \mathrm{~kg}$, $W L \%=34.9$ compared with the $U B W, B M I=14.6 \mathrm{~kg} /$ m2) (Fig. 1).

At the beginning of hospitalization, the patient received a specialized oral diet (low-fat, low insoluble fiber, free of lactose and sucrose, divided into seven to nine meals) ${ }^{9}$, either exclusively or in combination with a low-fat, high-protein semi-elemental enteral diet via a feeding tube positioned in the stomach ( $50 \%$ of daily energy requirement [DER]). On the 24 th day of admission, the patient had a clinical picture suggestive of partial occlusion of the bowel having been recommended observation of clinical course and a prescription for PN solution exclusively (nil per os). This regimen was maintained for 22 days, when a small volume of a semi-elemental diet was added via the feeding tube. After 30 days, PN was suspended, and a specialized oral diet was initiated?.

In December 2011, the patient was discharged. At that time, he presented six episodes of soft feces/ day, albumin $=2.34 \mathrm{~g} / \mathrm{dl}$, normal renal function, and $B W=51.2 \mathrm{~kg}$ (recovery of $6.5 \mathrm{~kg}$ ). At discharge, a specialized oral $\operatorname{diet}^{9}$ along with an enteral standard polymeric diet was prescribed.

In January 2012, the patient was readmitted for fluid and electrolyte imbalance $(\mathrm{Mg}=1.0 \mathrm{mg} / \mathrm{dl}, \mathrm{Na}=119$ $\mathrm{mEq} / \mathrm{l}$ ) and a weight loss of $4.4 \mathrm{~kg}$ in 33 days $(B W=46.8 \mathrm{~kg})$. During hospitalization, the patient had multiple episodes of sepsis with positive blood cultures and catheter tip positive for Staphylococcus epidermidis, Pseudomonas, Candida parapsilosis, Acinetobacter baumannii, gram-negative Rods. The patient was treated with Piperacillin + Tazobactam (1 4 days), Vancomycin (35 days), Fluconazole (28 days), Imipenem (34 days) Micafugin (26 days), Polymyxin (14 days), Cefepime (14 days) and Tigecycline ( 14 days).

Immediately after admission, a specialized oral diet $(70 \% \text { of DER })^{9}$ with an enteral standard polymeric diet $(30 \%$ of DER) was administered. On the 11 th day of admission, the patient received a clinical diagnosis of intestinal occlusion with the absence of stool elimination, abdominal distension, and vomiting. Oral and enteral diets were suspended. After a short period of observation without clinical improvement, 
the patient underwent exploratory laparotomy having been carried out lysis of adhesions. During surgical procedure was evidenced marked dilation of the remaining small intestine and stomach.

During the postoperative period, exclusive PN was administered, being observed a reduction in the number of evacuations and weight recovery (1.8 $\mathrm{kg})$. The PN solution was suspended on the 26th day because of liver complications [gamma-glutamyl transferase $(\circ \mathrm{GT})=552 \mathrm{U} / \mathrm{l}$; alkaline phosphatase $(\mathrm{AF})=666 \mathrm{U} / \mathrm{l}]$, and an exclusively enteral commercial semi-elemental diet was administered via a feeding tube. On the seventh day of this diet, the patient developed a new clinical picture suggestive of intestinal obstruction. Although subtle changes in the liver canalicular enzymes ( $\circ \mathrm{GT}=145 \mathrm{U} / \mathrm{l}, \mathrm{AF}=225$ $U / I)$ due to severe nutritional impairment and the inability to use the gastrointestinal tract for nutrition, were administered partial volumes of PN solutions with additional volumes of serum therapy to replace water and electrolytes.

During the reintroduction of enteral semi-elemental diet $(300 \mathrm{ml} /$ day $)$, was observed an increased frequency of evacuations (from four to nine stools/ day). Because of the advantages of administering diets via the gastrointestinal tract [2], particularly for patients with intestinal resection, new attempts were made using much smaller volumes of diet. The patient's tolerance of the semi-elemental diet was observed with the administration of $40 \mathrm{ml} /$ day $(5.0 \mathrm{ml} / \mathrm{h}$ for $8 \mathrm{~h}$ via a continuous infusion pump, with gastrointestinal tract rest for $16 \mathrm{~h}$ ). The volume of diet planned for the enteral route (50\% of DER) was reached after 18 days. Concurrent with the reintroduction of enteral feeding, the patient was given PN ( $50 \%$ of DER), intravenous fluids (for the additional replacement of fluid and electrolytes), and very small volumes of an oral clear liquid diet without sucrose one to two times/day.

With a partial improvement of diarrhea, the PN volume administered was gradually reduced until it was completely suspended. Further, the transition of the semi-elemental diet to a polymeric diet $(50 \%$ of DER) was carried out and the supply of orally administered food was increased. Oral foods were introduced in a gradual and evolutionary manner. Foods prepared without additional fat were introduced according to the following sequence: potatoes, rice, cornmeal, pasta, cassava, chayote, carrots, bananas, lean meat, cream crackers, watermelon, and cantaloupe. This approach was employed to ensure that each food was tolerated before a new food was administered. Every one or two days, the patient was offered a new food without excluding any previously tolerated food. Initially, the foods were given four times/day in small portions of approximately $120 \mathrm{~g} / \mathrm{meal}$ [9]. At discharge, the patient was following a scheduled diet of seven to eight meals/day.

After 132 days of hospitalization (June 2012), the patient was discharged with partial improvement of clinical status, fluid and electrolyte balance, weight recovery of $5.2 \mathrm{~kg}(B W=52 \mathrm{~kg}, W L \%=22.4$ compared with the $U B W, B M I=17.4 \mathrm{~kg} / \mathrm{m} 2)$, and the serum albumin of $3.2 \mathrm{~g} / \mathrm{dl}$.

Because of the great difficulty of obtaining and maintaining fluid and electrolyte balance and many acute renal failure episodes, the patient was instructed to consume four to six liters of fluid/day, including water, coconut water, and commercial rehydration solutions. Was also prescribed oral electrolytes replacement (magnesium sulfate $=160$ $\mathrm{mEq} /$ day; potassium chloride $=72 \mathrm{mEq} /$ day; sodium bicarbonate $=8$ teaspoons $/$ day). A medical doctor was responsible for monitoring the patient's serum electrolytes every two days and adjusted the intravenous fluid replacement and electrolytes doses when necessary.

As of January 2013, the patient was being followed at a multidisciplinary outpatient clinic. The patient had maintained fluid and electrolyte balance with preserved renal function and rarely required intravenous electrolytes correction (the last admission was in August 2012). The patient had significant weight recovery $(B W=60.2 \mathrm{~kg}, B M I=20.1 \mathrm{~kg} / \mathrm{m} 2$, a recovery of $16.6 \mathrm{~kg}$ ) (Fig. 1) but exhibited a BW deficit compared with the normal BW (WL\% $=10.2)$. There has been an improvement in the pattern of bowel movements (three to four small, formed stools/day) and a recovery of functional capacity, including the capacity to work. The patient consumed an oral diet with his family; his diet had a normal consistency and was restricted only in terms of excess lipids, insoluble fiber, lactose, and sucrose.

Definitive discharge of the outpatient clinic was 
performed in July 2014, after electrolytes supplementation withdrawal and recovery of regular body weight $(B W=67.2 \mathrm{~kg})$.

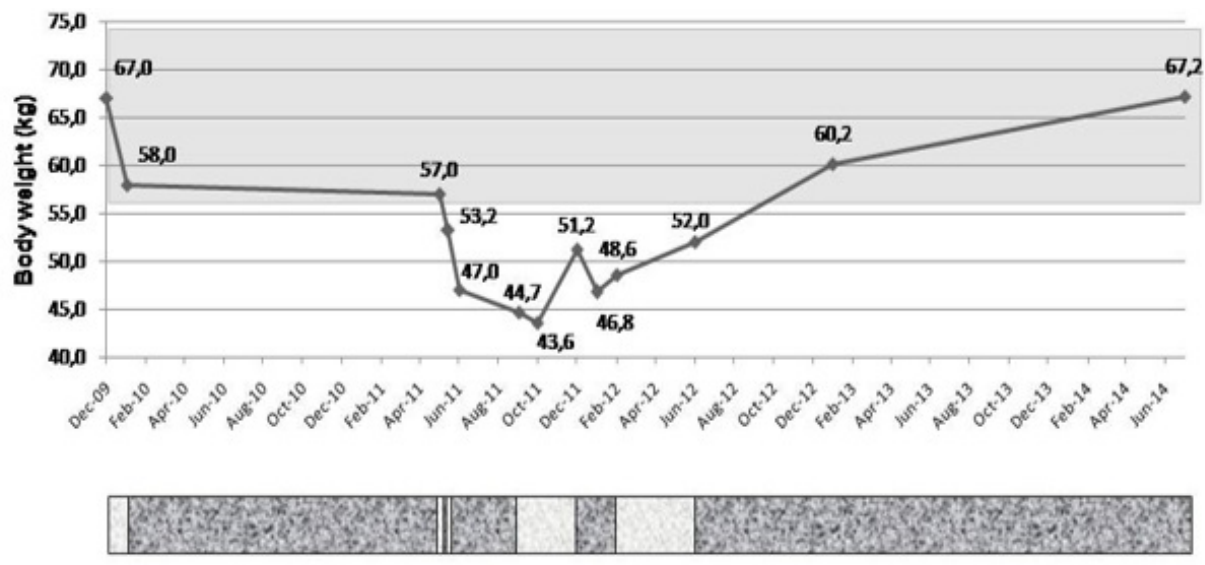

\section{Legend:}

Body mass index (BMI) range corresponding to eutrophic nutritional status for the patient's age (BMl=18.5 to $24.99 \mathrm{~kg} / \mathrm{m}^{2}$ )

Hospitalization

Outpatient follow-up

\section{DISCUSSION}

In the present study, we report the clinical course of a patient with a severe intestinal failure associated with total colectomy and partial ileectomy (removal of $60 \mathrm{~cm}$ of the terminal ileum and the ileocecal valve) with ileorectal anastomosis. Postoperatively, the patient developed severe protein-energy malnutrition, acute renal failure, and a fluid and electrolyte disturbance that was marked, prolonged, and very difficult to control.

The high frequency of bacteremia and sepsis presented by the patient may be related to multiple causes. In addition to the high frequency of systemic infections associated with prolonged use of parenteral nutrition solutions, and the changes in the intestinal microbiome of patients with short bowel syndrome $^{10}$, one must consider that the patient had been diagnosed with Chagas' disease. Clinical manifestations in small intestine in patient's Chagas' disease, are characterized by abnormalities of motility and dilation, such as mega-duodenum and mega-jejunum. Bacterial overgrowth in the dilated small intestine in Chagas' disease has been associated with fast transit, chronic diarrhea, malabsorption, and steatorrhea ${ }^{11}$. In addition, bacterial overgrowth is a strong causal factor for bacterial translocation and development of systemic infections or sepsis ${ }^{12}$.

Patient's severe protein-energy malnutrition, inducing a clinical status compatible with secondary immunodeficiency, may also have been a significant causal factor in the high frequency of bacteremia and sepsis. Malnourished patients presented systemic infections characterized by atypical clinical manifestations (e.g., the absence of fever or other clinical manifestations in the presence of positive blood cultures) and a poor response to antibiotic treatment ${ }^{13}$, both presented by the patient. These considerations are consistent with the results of a study recently published. Using a logistic model, the investigators demonstrated that malnourished patients had a significantly increased risk of developing infectious complications compared with well-nourished patients (odds ratio $=19.80)^{14}$.

Established guidelines state that SBS patients with higher nutritional risks are those with intestinal failure associated with resection of the duodenum 
or jejunoileal anastomosis and $<35 \mathrm{~cm}$ of remaining small intestine, jejunal resection with $<115 \mathrm{~cm}$ of remaining small intestine, or jejunocolic or ileocolic anastomosis with $<60 \mathrm{~cm}$ of remaining small intestine [6]. In the clinical case presented in this study, the patient had severe protein-energy malnutrition (WL\%=34.9 compared with the UBW), although he did not meet any of the conditions listed above.

The long period that elapsed (55 months) before the development of intestinal adaptation by this patient was possibly associated with chronic gastrointestinal manifestations of Chagas disease, characterized by a marked and diffuse dilatation of the remaining small intestine and stomach, and a large number of adhesions between intestinal loops. These changes caused episodes of partial or complete intestinal obstruction and volvulus of the stomach, resulting in periods of fasting and worsening protein-energy malnutrition. In addition, patients without an intact colon exhibit impaired water absorption and lower stimulus for hyperplasia of the small intestine ${ }^{7}$.

After the resection of intestinal segments, the body develops adaptive mechanisms in the remaining intestine in an attempt to regain the ability to absorb nutrients ${ }^{15}$. The presence of nutrients in the intestinal lumen (luminal factor) is essential in the process of adaptive mucosal hyperplasia and intestinal adaptation $^{2}$. In the case of the patient reported in this study, after several months a severe intestinal failure was controlled by administering very small volumes of a semi-elemental diet and a specialized oral diet concomitantly with PN solutions.

Among the factors associated with the prognosis of patients with SBS and intestinal insufficiency are the length and the segment of the remaining small intestine, the absence of underlying diseases, the presence of the colon and ileocecal valve, and the causal factor of the SBS. Prognostic factors also include the patient's age and the state of intestinal adaptation $^{6}$. Although the patient reported in this study presented several poor prognostic factors, the use of the reported therapeutic and nutritional regimens enabled the recovery of his nutritional status and functional capacity.

\section{CONCLUSION}

Independently of the absence of high output ostomy, patients undergoing total colectomy and partial ileectomy resection can be present severe fluid and electrolyte imbalance, protein-energy malnutrition, and systemic infectious diseases. In this sense the development of intestinal adaptation is essential for homeostasis / metabolic control in patients undergoing resection of specific intestinal segments (colon, terminal ileum and the ileocecal valve), particularly for those patients who had alterations of the anatomical structure of the gastrointestinal tract, associated for example, with the chronic evolution of Chagas' disease.

The involvement of an updated, cohesive multidisciplinary team that is committed to the patient's clinical outcome, assessing pathophysiological changes and adapting the patient's nutritional protocol to his/her clinical needs is essential for meeting the challenge of recovering the nutritional status of patients with intestinal failure associated with functional SBS.

\section{ACKNOWLEDGEMENTS}

We would like to thank the Multidisciplinary Residency Program in Health Pharmaceutical Members Marcia R. Bortolini and Mariana D. Góes for their cooperation in outpatient and hospital care provided to the patient. We would like to thank the pharmaceutical Rita A. Cardoso, MSc, Professor of the Triangle University Center, Uberlandia, MG, Brazil.

\section{AUTHOR CONTRIBUTIONS}

Duarte TC and De-Souza DA participated in the planning and design of the study, carried out the study, interpreted the data and elaborated the manuscript. Melo FG participated in the planning and design of the study and helped in drafting the initial version of the manuscript. Santos-Júnior JR, Moussa MP helped in data collection, interpreted the work data and contributed in drafting the initial version of the manuscript. All authors have read and approved the final version of the manuscript. All authors agree upon accountability concerning all aspects of the work in ensuring that questions related to the accuracy or integrity of any part of the work are appropriately investigated and resolved.

\section{COMPETING INTERESTS}

No financial, legal or political competing interests with third parties (government, commercial, private foundation, etc.) were disclosed for any aspect of the submitted work (including but not limited to grants, data monitoring board, study design, manuscript preparation, statistical analysis, etc.). 


\section{REFERENCES}

1. Pironi L, Arends J, Baxter J, Bozzetti F, Pelaez RB, Cuerda $C$ et al. ESPEN endorsed recommendations. Definition and classification of intestinal failure in adults. Clin Nutr. 2015;34(2):171-180. doi: 10.1016/i.clnu.2014.08.017

2. ASPEN Short bowel syndrome: clinical guidelines for nutrition management. Nutr Clin Pract. 2005;20(5):493-502

3. O'Keefe SJD, Buchman AL, Fishbein TM, Jeejeebhoy KN, Jeppesen PB, Shaffer J. Short bowel syndrome and intestinal failure: consensus definitions and overview. Clin Gastroenterol Hepatol. 2006;4(1):6-10. doi: 10.1016/i.cgh.2005.10.002

4. De-Souza DA, Greene, LJ. Nutritional issues in obese patients submitted to bariatric surgery. Re." Bariatric surgery may not achieve intended outcomes in all patients". Nutrition. 2015;31(9):1 184-1 185. doi: 10.1016/i.nut.2015.06.003

5. Tappenden KA. Pathophysiology of short bowel syndrome: considerations of resected and residual anatomy. J Parenter Enteral Nutr. 2014;38(1 Suppl):14S-22S. doi: $\underline{10.1177 / 0148607113520005}$

6. AGA: American Gastroenterological Association medical position statement. Short bowel syndrome and intestinal transplantation. Gastroenterology. 2003;1 24(4):1 105-1 110

7. Nordgaard I, Hansen BS, Mortensen PB. Importance of colonic support for energy absorption as small-bowel failure proceeds. Am J Clin Nutr. 1996;64(2):222-231

8. Straatman J, Cuesta MA, de Lange - de Klerk ESM, van der Peet DL. Long-term survival after complications following major abdominal surgery. J. Gastrointest Surg. 2016;20:1034-1041. doi: 10.1007/s1 1605-016-3084-4

9. Lopes MGF, De-Freitas LA, Martins TCP, Mosca ERT, Silva AASC, De-Souza DA. Specialized oral diet improved the clinical outcome of a patient with severe intestinal insufficiency in a late postoperative period. A case report in clinical nutrition. J Acad Nutr Diet. 2016;1 16:1 243-1 250. doi: 10.1016/i.jand.2016.03.002

10. Davidovics ZH, Carter BA, Luna RA, Hollister $E B$, Shulman RJ, Versalovic J. The fecal microbiome in pediatric patients with short bowel syndrome. J Parenter Enteral Nutr. 2015;40(8):1 106-1 113 . doi: $\underline{10.1177 / 0148607115591216}$

11. Matsuda NM, Miller SM, Évora PRB. The chronic gastrointestinal manifestations of Chagas disease. Clinics. 2009;64(1 2): $1219-1224$. doi: 10.1590/S1807$\underline{59322009001200013}$

12. De-Souza DA, Greene J. Intestinal permeability and systemic infections in critically ill patients. Effect of glutamine. Crit Care Med. 2005;33(5): $1125-1135$
13. Chandra RK. Nutrition and the immune system: an introduction. Am J Clin Nutr. 1997;66(2):460S-463S

14. Pasquini TAS, Neder HD, Araújo-Junqueira L, De-Souza

DA. Clinical outcome of protein-energy malnourished patients in a Brazilian university hospital. Braz J Med Biol Res. 2012;45(12):1301-1307. doi: 10.1590/1414$\underline{431 \times 20122586}$

15. Nightingale JMD, Woodward JM. Guidelines for management of patients with a short bowel. Gut. 2006;55:1 12. doi: $10.1136 /$ gut. 2006.091108 